\title{
Distributing Antidote Using PageRank Vectors
}

\author{
Fan Chung, Paul Horn, and Alexander Tsiatas
}

Abstract. We give an analysis of a variant of the contact process on finite graphs, allowing for nonuniform cure rates, modeling antidote distribution. We examine an inoculation scheme using PageRank vectors that quantify the correlations among vertices in the contact graph. We show that for a contact graph on $n$ nodes we can select a set $H$ of nodes to inoculate such that with probability at least $1-2 \epsilon$, any infection from any starting infected set of $s$ nodes will die out in $c \log s+c^{\prime}$ time, where $c$ and $c^{\prime}$ depend only on the probabilistic error bound $\epsilon$ and the infection rate, and the size of $H$ depends only on $s, \epsilon$, and the topology around the initially infected nodes, independent of the size of the whole graph.

\section{Introduction}

The spreading and containment of epidemics on networks is a widely studied problem with many applications in modeling disease outbreaks in both human and animal populations as well as the spread of viruses and worms on technological networks such as the Internet, online social networks, and email. Many analytical models have been used to address numerous crucial problems, such as the conditions for spread of disease, the critical threshold for the infection rate, the duration of persistent epidemics, and the effective distribution of limited amounts of antidote. We will examine a well-studied contact process model [Borgs et al. 10, Newman 02], coupled with an inoculation scheme using PageRank vectors. In this paper, we give a complete analysis of our scheme, showing

(C) A K Peters, Ltd. 
the improved efficiency of the inoculation scheme without affecting the performance guarantee as in previous results in [Borgs et al. 10].

A contact graph consists of a set of nodes together with prescribed pairs of nodes where direct contact can take place and infections can spread (see [Chen and Carley 03, Ganesh et al. 05, Newman 02]). Analysis of spreading on the contact graph is performed with the contact process, a continuous-time Markov process originally studied in the first half of the twentieth century [Kermack and McKendric 27]. Since then, it has been applied specifically to network epidemics in many contexts, including social networks [Tsimring and Huerta 03], Internet viruses [Beger et al. 05], and crop disease [Forster and Gilligan 07].

Previously, most analysis of network infection models concerned determining the critical infection threshold [Beger et al. 05, Ganesh et al. 05, Newman 02]. There is a parameter, known as the infection rate, that models the virulence or resistance of a given epidemic, and with it comes a threshold: if the infection rate exceeds that point, then an epidemic will persist indefinitely. In these analyses, the infected nodes became healthy all at the same rate. In the contact process, this occurs when an equal amount of antidote is sent indiscriminately to all nodes, requiring a large amount of antidote. In practice, this is often undesirable; in this paper, we will give a model that avoids such widespread antidote distribution.

Another approach is to combat epidemics using contact tracing, or inoculating neighbors of infected nodes, using a total amount of antidote that depends only on the sum of the degrees of the infected nodes. However, both simulation and mathematical analysis have shown that contact tracing can be ineffective, especially on large real-world graphs that exhibit small-world phenomena and power-law degree distributions [Dezso and Barabasi 02, Kiss et al. 05, May and Lloyd 01, Pastor-Sattoras and Vespignani 01, Pastor-Sattoras and Vespignani 02, Tsimring and Huerta 03].

In [Borgs et al. 10], the authors show that for the contact process on a contact graph $G$, inoculating every node with antidote equal to its degree will result in any infection dying out in $O(\log n)$ time with high probability, where $n$ is the number of nodes in $G$. This scheme uses a total amount of antidote equal to the sum of the degrees in $G$. For special graphs such as the expanders, it has been shown [Borgs et al. 10] that such a large amount of antidote is necessary (up to a constant factor) when a constant proportion of the nodes are initially infected. Our proposed model will not improve this result on expander graphs, but for many classes of graphs, we will require antidote only on smaller portions of the network.

In this paper, we analyze an inoculation scheme using PageRank vectors. PageRank was first introduced in [Brin and Page 98] for web search algorithms. Although the original definition is for the Web graph, PageRank is well defined 
for any graph, including the contact graphs that we study. Here, we will use a modified version of PageRank, known as personalized PageRank, for the contact graph with the initially infected nodes as seeds. PageRank captures the quantitative correlation between pairs or subsets of nodes. For example, if the contact graph has some small cuts or bottlenecks, it is likely that an infection will not propagate through them, and nodes on the other side will have low PageRank. Our inoculation scheme using PageRank specifies the selected nodes for sending antidote and provides a probabilistic guarantee for the termination of any epidemic. Furthermore, the number of selected nodes for inoculation is usually much smaller than the total size of the contact graph, thus improving the previous schemes that inoculated all the nodes. The number of selected nodes depends only on the number of initially infected nodes, the probabilistic guarantee bound, and the isoperimetric invariant of the graph: the Cheeger ratio (to be defined later). Hence, it is independent of the total size of the contact network.

Previously, an empirical study [Miller and Hyman 07] found that inoculating nodes according to their PageRank works well in combating epidemics for certain examples of contact networks. Our analysis complements this experimental work and is applicable to any given general contact network. The analysis in Section 3 provides a tradeoff between the probabilistic guarantee of termination and the time required.

\section{Preliminaries}

We model an epidemic spreading on a general undirected contact graph $G=$ $(V, E)$ with vertex set $V$ and edge set $E$. For a vertex $v$, let $d_{v}$ denote the degree of $v$, which is the number of neighbors of $v$. Suppose that the graph $G$ has $n$ nodes with a degree sequence $\mathbf{d}=\left(d_{1}, d_{2}, \ldots, d_{n}\right)$, where $d_{i}$ is the degree of vertex $v_{i}$. For a set of nodes $T \subseteq V$, the volume of $T$ is defined to be $\operatorname{vol}(T)=\sum_{v \in T} d_{v}$. Let $D$ denote the diagonal degree matrix $\operatorname{diag}\left(d_{1}, \ldots, d_{n}\right)$ and $A$ the adjacency matrix of $G$, where

$$
A_{i j}= \begin{cases}1 & \text { if }\left\{v_{i}, v_{j}\right\} \in E \\ 0 & \text { otherwise }\end{cases}
$$

We consider a typical random walk on $G$ with the transition probability matrix defined by $W=D^{-1} A$. Personalized PageRank vectors are based on random walks and $W$, with two governing parameters: a seed vector s, representing an initial distribution over $V$, and a jumping constant $\alpha$, which controls the rate of diffusion. Since $\mathbf{s}$ is a distribution, its entries sum to 1 . The personalized 
PageRank $\operatorname{pr}(\alpha, \mathbf{s})$ is defined to be the solution to the following recurrence relation:

$$
\operatorname{pr}(\alpha, \mathbf{s})=\alpha \mathbf{s}+(1-\alpha) \operatorname{pr}(\alpha, \mathbf{s}) W .
$$

Here, s (and all other vectors) will be treated as row vectors. The original definition of PageRank defined in [Brin and Page 98] is the special case in which the seed vector is the uniform distribution (as used in [Miller and Hyman 07]).

From (2.1), an alternative expression for the personalized PageRank $\operatorname{pr}(\alpha, \mathbf{s})$ is a geometric sum of random walks (see [Andersen et al. 06]):

$$
\operatorname{pr}(\alpha, \mathbf{s})=\alpha \sum_{t=0}^{\infty}(1-\alpha)^{t} \mathbf{s} W^{t} .
$$

For a subset of nodes $H$ in a graph $G$, the Cheeger ratio $h(H)$ is a measure of the cut between $H$ and its complement $\bar{H}$ :

$$
h(H)=\frac{e(H, \bar{H})}{\min (\operatorname{vol}(H), \operatorname{vol}(\bar{H}))},
$$

where $e(H, \bar{H})$ denotes the number of edges $\{u, v\}$ with $u \in H$ and $v \in \bar{H}$. For a given value $h$, we say that $H$ is an $h$-cluster if its Cheeger ratio $h(H)$ satisfies $h(H) \leq h$.

For an $h$-cluster $H$ and a given $\alpha$, the $\alpha$-core $C$ of $H$ is the set of all vertices $u$ such that the personalized PageRank on $H$, with seed $u$ and jumping constant $\alpha$, is at least $1-\frac{h}{\alpha}$ :

$$
C=\left\{u \in V \mid \operatorname{pr}\left(\alpha, \mathbf{1}_{u}^{*}\right) \mathbf{1}_{H} \geq 1-\frac{h}{\alpha}\right\} .
$$

It has been shown [Andersen et al. 06] that if $C$ is the $\alpha$-core of $H$, then $\operatorname{vol}(C) \geq \frac{1}{2} \operatorname{vol}(H)$. This indicates that there are many nodes $u \in H$ for which the personalized PageRank vector $\operatorname{pr}\left(\alpha, \mathbf{1}_{u}^{*}\right)$ has very little mass outside of $H$ if $\alpha$ is larger than $h$.

\section{Infection Model and Inoculation Scheme}

We use the following contact process (see [Kermack and McKendric 27]) as our infection model, which is also used in [Beger et al. 05]. The contact process is a continuous-time Markov process parameterized by $\beta$, the infection rate, with $0 \leq \beta<1$ and $\mathbf{c}=\left(c_{1}, c_{2}, \ldots, c_{n}\right)$, the cure vector. We assume that at time 
$t=0$, a seed set $S \subseteq V$ is infected. We will use $\mathbf{1}_{S}$ to denote the indicative vector associated with $S$, and $\mathrm{x}(0)=\mathbf{1}_{S}$.

Each node $v_{i}$ has an infection state $x_{i}(t)$; a node is considered "healthy" if $x_{i}(t)=0$, and "infected" if $x_{i}(t)=1$. Thus, the entire process is characterized by a state vector $\mathbf{x}(t)=\left(x_{1}(t), x_{2}(t), \ldots, x_{n}(t)\right)$. The state transitions are as follows:

- If a node $x_{j}$ is infected, an adjacent node $x_{i}$ becomes infected at rate $\beta$. We refer to this transition as a spread event.

- An infected node $v_{i}$ becomes healthy at rate $c_{i}$. We refer to this transition as a cure event.

In any continuous-time Markov process, for a transition (e.g., spread or cure event) that occurs with rate $\lambda$, the elapsed time until that transition takes place assumes an exponential random variable with parameter $\lambda$, which is independent of any state information. Such a random variable has a probability density function $f(x)=\lambda e^{-\lambda x}$ for $x \geq 0$, and 0 otherwise. We denote such a random variable by $\operatorname{Expo}(\lambda)$.

Using this contact process as a model, our goal is to choose $\mathbf{c}$ such that with high probability, the infection dies out quickly, and the total amount of antidote used is small. Furthermore, we want $\mathbf{c}$ to depend only on the seed set $S$ and the degree distribution $\mathbf{d}$, but not on $t$ or $\mathbf{x}(t)$. Our main theorem describes how to find such a cure vector $\mathbf{c}$. First, we establish a relationship between PageRank and the infection starting from $S$ but leaving a specified area.

Theorem 3.I. Suppose that an infection starts in $S \subseteq H \subseteq V$ with infection rate $\beta$, and each node $v \in H$ is inoculated with $c_{v}=d_{v}$. Let $\mathcal{E}_{H}$ denote the event that an infection started in $S$ ever leaves the set $H$. Then $\mathcal{E}_{H}$ can be bounded above by the PageRank vector as follows:

$$
\mathbf{P}\left(\mathcal{E}_{H}\right) \leq \frac{s}{\beta} \operatorname{pr}\left(1-\beta, \frac{\mathbf{1}_{S}}{s}\right) \mathbf{1}_{\bar{H}}^{*}
$$

The proof of Theorem 3.1 will be given in Section 5. Using this theorem, we can further derive the following:

Theorem 3.2. Let $G$ be a contact graph with $n$ nodes, $S$ an initial set of infected nodes with $|S|=s$, and $\beta$ the infection rate with $0 \leq \beta<1$. Suppose that $H$ is an $h$-cluster that contains $S$ in its $(1-\beta)$-core. If all nodes in $H$ are inoculated with antidote equal to their degrees, then with probability at least $1-2 \frac{s h}{\beta(1-\beta)}$, 
Algorithm I. (InoculationScheme $(G, S, \beta, \epsilon)$ )

Input: A contact graph $G$, an initial set $S$ of $s$ infected nodes, the infection rate $\beta$, and the error bound $\epsilon$

1. Set $h=\frac{\epsilon}{s} \beta(1-\beta)$.

2. Use [Andersen et al. 06, PageRank-Nibble] or [Andersen and Chung 07, Local Partition] to find an $h$-cluster $H$ containing $S$, if one exists.

3. Check to see whether $S$ is in the $(1-\beta)$-core of $H$. If so, then inoculate each node $v \in H$ with $c_{v}=d_{v}$.

4. If $S$ is not in the core of $H$, or an $h$-cluster could not be found, then let $H=G$ and inoculate every node with $c_{v}=d_{v}$.

any infection starting from $S$ will die out in at most $c \log (1 / h)+c^{\prime}$ time, where $c$ and $c^{\prime}$ depend only on $\beta$ and not on $n$.

Theorem 3.2 will be proved in Section 5.

We remark that Theorem 3.2 implies a tradeoff between the Cheeger ratio $h$ and the probabilistic bound. If the initial set of infected nodes $S$ lies within the $(1-\beta)$-core of an $h$-cluster $H$, the probability of the infection dying out in $O(\log s)$ time is high, as long as the product $s h$ is small. In particular, if the seed set $S$ lies on one side of a small cut, it will likely lie within the core of an $h$-cluster with small Cheeger ratio $h$. If there is no such small cut, then the infection is likely to spread about the graph. This leads to the following corollary.

Corollary 3.3. For any $\epsilon>0$ and an infection starting from a seed set $S$, if $S$ lies within the $(1-\beta)$-core of an h-cluster $H$, and $h \leq \frac{\epsilon}{s} \beta(1-\beta)$, then with probability at least $1-2 \epsilon$, the infection will die out in $c \log s+c^{\prime}$ time, where $c$ and $c^{\prime}$ depend only on $\beta$ and $\epsilon$ and not on $n$.

The proof of the corollary follows from applying the bound on $\epsilon$ to Theorem 3.2. We note that the above corollary explicitly relates the desired probabilistic guarantee $\epsilon$ to the Cheeger ratio $h$ of the $h$-cluster containing $S$ in its core.

The above theorems suggest the inoculation scheme of Algorithm 1. This inoculation scheme relies on the ability to find a cluster $H$ that has small Cheeger ratio $h$ and contains $S$ in its core. If such an $H$ does not exist, then the algorithm will terminate with the entire graph inoculated. For example, in the case of 
expander graphs, the algorithm will soon terminate and is reduced to the same scenario as in [Borgs et al. 10]. Nevertheless, it is likely for a general contact graph to contain small $h$-clusters. In such cases, only a small portion of the graph needs to be inoculated, while the desired performance guarantee is maintained.

Next, we consider the case that the initially infected nodes are randomly distributed in an $h$-cluster $H$. We might expect that the infection is not likely to escape $H$. This is not strictly true, because if $S$ contains some nodes near the boundary of $H$, it is still quite likely that the infection will escape. Nevertheless, we will be able to establish an upper bound for such probability by proving the following theorem:

Theorem 3.4. Suppose $H \subseteq G$ is an h-cluster, and the set $S$ of initially infected nodes consists of $s$ nodes randomly and independently selected from $H$ with probability proportional to their degrees. Suppose the infection rate is $\beta$. Then, for a given $\epsilon$ satisfying sh $\leq \epsilon$ and $s \geq \log (1 / \epsilon) / \epsilon$, if all nodes in $H$ are inoculated with antidote equal to their degrees, then with probability at least $1-\epsilon$, any infection starting from $S$ will die out in $c \log s+c^{\prime}$ time, where $c$ and $c^{\prime}$ depend only on $\epsilon$ and $\beta$.

The proof of Theorem 3.4 will be given in Section 5. This randomized model is relevant when a disease outbreak originates in a subpopulation, which can be represented as an $h$-cluster in a larger population graph. Theorem 3.4 implies that if the subpopulation is relatively isolated from the rest of the population (i.e., the Cheeger ratio $h$ is small), then we can effectively combat the infection by attacking the epidemic only within that $h$-cluster.

\section{An Outline of the Analysis of the Inoculation Scheme and Several Useful Facts}

In order to prove Theorems 3.1 and 3.2, we will prove several basic facts in the following brief outline of our analysis of the inoculation scheme:

- The probability of the infection spreading to a distant node is small (Lemma 4.1).

- The probability that a nearby node will remain infected for a long time is small (Lemmas 4.2, 4.3).

- The probability that an infection persists within the inoculated nodes is small (Lemma 4.4). 
- The probability that an infection escapes the inoculated nodes depends on the PageRank on the uninoculated nodes $\bar{H}$, proving Theorem 3.1.

- After Theorem 3.1 has been proved, Theorem 3.2 follows from the fact that the personalized PageRank on $\bar{H}$ is small.

In this section, we will first give some definitions and then proceed to prove Lemmas 4.1-4.4.

Consider that if a vertex $v_{k}$ is infected at some time $t$, then the infection must have traversed some walk in the graph from a vertex $v_{0} \in S$. Suppose $\pi=\left(v_{0}, \ldots, v_{k}\right)$ is a path in $G$ of length $k$. Let $\mathcal{S}_{\pi}$ denote the event that $v_{0}$ is infected at time 0 , and the infection spreads to $v_{k}$ before time $t$ along the path $\pi$. It is important to note that if $\mathcal{S}_{\pi}$ occurs, $v_{k}$ is not necessarily infected at time $t$, because it could have been cured before time $t$; however, $v_{k}$ cannot be infected at time $t$ if no $\mathcal{S}_{\pi}$ occurred.

For a vertex $v$, let $C(v, t)$ denote the time of the first cure event at vertex $v$ after time $t$. From this, we define a realization of a walk as the sequence of random variables $X_{v}$ as follows:

- A spread event from vertex $v_{i}$ to $v_{i+1}$ occurs at time $X_{i+1}$.

- $0<X_{1}<C\left(v_{0}, 0\right)$.

- For all $i \geq 1, X_{i}<X_{i+1}<C\left(v_{i}, X_{i}\right)$.

For $\mathcal{S}_{\pi}$ to occur, the infection must spread to $v_{k}$ before time $t$; therefore, $\mathcal{S}_{\pi}$ occurs if and only if there is a realization of $\pi$ with $X_{k}<t$. There are many possible realizations of $\pi$, but in our analysis, we will be concerned with a specific realization: the canonical realization. In this realization, given the times of all the cure and spread events, $X_{i}$ is the maximum over all possible realizations with those cure and spread times. Thus, the canonical realization is the latest possible infection path along $\pi$.

With $X_{i}$ as in the canonical realization, we also define an event $\mathcal{S}_{\pi}^{\prime}$ that occurs when at least one spread event from $v_{i}$ to $v_{i+1}$ occurs between $X_{i}$ and $C\left(v_{i}, X_{i}\right)$. Thus, if $\mathcal{S}_{\pi}^{\prime}$ occurs, then the infection spreads along $\pi$ to $v_{k}$, but not necessarily before time $t$. While we are primarily concerned with the event $\mathcal{S}_{\pi}$. When the spread occurs before time $t$, it is clear that $\mathcal{S}_{\pi} \subseteq \mathcal{S}_{\pi}^{\prime}$, and using the canonical realization allows us to prove the following lemma, which indicates that the probability that an infection follows a long path is small: 
Lemma 4.I. For any path $\pi$ of length $k$,

$$
\mathbf{P}\left(\mathcal{S}_{\pi}\right) \leq \mathbf{P}\left(\mathcal{S}_{\pi}^{\prime}\right) \leq \beta^{k} \prod_{j=0}^{k-1} \frac{1}{c_{j}}
$$

Proof. Let $\mathcal{S}_{j}$ denote the event that there is a spread event from $v_{j}$ to $v_{j+1}$ between times $X_{j}$ and $C\left(v_{j}, X_{i}\right)$. Due to the Markov property of the contact process, the probability of $\mathcal{S}_{j}$ occurring is

$$
\mathbf{P}\left(\mathcal{S}_{j}\right) \leq \frac{\beta}{c_{j}} .
$$

Since the curing process at every node is independent, we can write

$$
\mathbf{P}\left(\mathcal{S}_{\pi}\right) \leq \mathbf{P}\left(\mathcal{S}_{\pi}^{\prime}\right)=\prod_{j=0}^{k-1} \mathbf{P}\left(\mathcal{S}_{j}\right)=\beta^{k} \prod_{j=0}^{k-1} \frac{1}{c_{j}}
$$

For a walk $\pi$ of length $k$ with canonical realization $\left(X_{i}\right)_{i=1}^{k}$, we define the canonical end time of $\pi$ to be

$$
Z_{\pi}= \begin{cases}X_{k} & \text { if } \mathcal{S}_{\pi} \text { occurs } \\ 0 & \text { otherwise }\end{cases}
$$

In other words, $Z_{\pi}$ is the last time that $v_{k}$ could become infected via the path $\pi$, or 0 if it is never infected via $\pi$. The following lemma states that the probability that $Z_{\pi}$ is large is small:

Lemma 4.2. Suppose for a path $\pi$ of length $k$, that $Z_{\pi}$ is its canonical end time. Then

$$
\mathbf{P}\left(Z_{\pi}>t\right) \leq \frac{1}{(2 k) !} t^{2 k-1} e^{-\beta t} \beta^{k} \prod_{j=0}^{k-1} \frac{1}{c_{j}} .
$$

Proof. Let $\left(X_{i}\right)_{i=1}^{k}$ denote the canonical realization of $\pi$. Then

$$
\begin{aligned}
\mathbf{P}\left(Z_{\pi}>t\right) & =\mathbf{P}\left(Z_{\pi}>t, \mathcal{S}_{\pi}\right) \\
& =\mathbf{P}\left(X_{k}>t, \mathcal{S}_{\pi}\right) \\
& \leq \mathbf{P}\left(X_{k}>t, \mathcal{S}_{\pi}^{\prime}\right) \\
& =\mathbf{P}\left(X_{k}>t \mid \mathcal{S}_{\pi}^{\prime}\right) \mathbf{P}\left(\mathcal{S}_{\pi}^{\prime}\right) .
\end{aligned}
$$


Applying Lemma 4.1, we have

$$
\mathbf{P}\left(Z_{\pi}>t\right) \leq \mathbf{P}\left(X_{k}>t \mid \mathcal{S}_{\pi}^{\prime}\right) \beta^{k} \prod_{j=0}^{k-1} \frac{1}{c_{j}}
$$

We further observe that

$$
\begin{aligned}
\mathbf{P}\left(X_{k-1}>t \mid \mathcal{S}_{\pi}^{\prime}\right) & =\mathbf{P}\left(\sum_{i=1}^{k}\left(X_{i}-X_{i-1}\right)>t \mid \mathcal{S}_{\pi}^{\prime}\right) \\
& \leq \mathbf{P}\left(\sum_{i=1}^{k}\left(C\left(v_{i}, X_{i-1}\right)-X_{i-1}\right)>t \mid \mathcal{S}_{\pi}^{\prime}\right) .
\end{aligned}
$$

We consider the time between $X_{i-1}$ and the first cure event at $v_{i}$ after $X_{i-1}$ subject to the condition $\mathcal{S}_{\pi}^{\prime}$ : at least one spread event occurred before the cure at $C\left(v_{i}, X_{i-1}\right)$. Therefore, the time between $X_{i-1}$ and $C\left(v_{i}, X_{i-1}\right)$ is at least the time for one spread event, namely, the exponential random variable $\operatorname{Expo}(\beta)$, plus the time for one cure event, $\operatorname{Expo}\left(c_{i}\right)$. Thus, we have

$$
\sum_{i=1}^{k}\left(C\left(v_{i}, X_{i-1}\right)-X_{i-1}\right) \geq \sum_{i=1}^{k}\left(\operatorname{Expo}(\beta)+\operatorname{Expo}\left(c_{i}\right)\right) .
$$

Because $\beta \leq c_{i}$, it follows that $\operatorname{Expo}\left(c_{i}\right)$ is stochastically dominated by $\operatorname{Expo}(\beta)$. We can write

$$
\sum_{i=1}^{k}\left(C\left(v_{i}, X_{i-1}\right)-X_{i-1}\right) \geq \sum_{i=1}^{k}(\operatorname{Expo}(\beta)+\operatorname{Expo}(\beta)) .
$$

The sum of $2 k$ independent exponential random variables has a gamma distribution $\Gamma(2 k, \beta)$. Therefore,

$$
\mathbf{P}\left(X_{k-1}>t \mid \mathcal{S}_{\pi}^{\prime}\right) \leq \frac{1}{(2 k) !} \int_{t}^{\infty} x^{2 k-1} e^{-x} d x \leq \frac{t^{2 k-1} e^{-\beta t}}{(2 k) !} .
$$

Putting all of this together, the lemma immediately follows.

The next lemma addresses the question whether a vertex $v$ is infected at time $t$. Note that $\mathcal{S}_{\pi}$ addresses only whether a vertex was infected via $\pi$ at some time before $t$; it could be cured thereafter.

If $\left(X_{i}\right)_{i=1}^{k}$ is the canonical realization of $\pi$, then we say that $v$ is infected at time $t$ via path $\pi$ if $\mathcal{S}_{\pi}$ occurs and the first cure event at $v_{k}$ after $X_{k}$ does not occur until after time $t$. We denote this event by $\mathcal{T}_{\pi, t}=\mathcal{S}_{\pi} \cap\left\{C\left(v_{k}, X_{k}\right)>t\right\}$. 
Lemma 4.3. Suppose $\pi$ is a walk of length $k$, and the amount of antidote at $v_{k}$ is $c_{k} \geq \beta$. Then

$$
\mathbf{P}\left(\mathcal{I}_{\pi, t}\right)<e^{-\beta t / 2}\left(1+\frac{1}{(2 k) !}(t / 2)^{2 k-1}\right) \beta^{k} \prod_{j=0}^{k-1} \frac{1}{c_{j}}
$$

Proof. We first note that the elapsed time from $X_{k}$ to the cure event $C\left(v_{k}, X_{k}\right)$ is an exponential random variable with parameter $c_{k}$, independent of $\mathcal{S}_{\pi}$. Thus, we can write

$$
\begin{aligned}
\mathbf{P}\left(\mathcal{T}_{\pi, t}\right) & =\mathbf{P}\left(C\left(v_{k}, X_{k}\right)>t, \mathcal{S}_{\pi}\right) \\
& =\mathbf{P}\left(\operatorname{Expo}\left(c_{k}\right)>t-X_{k}, \mathcal{S}_{\pi}\right) \\
& \leq \mathbf{P}\left(\operatorname{Expo}\left(c_{k}\right)>t / 2, \mathcal{S}_{\pi}, X_{k} \leq t / 2\right)+\mathbf{P}\left(X_{k}>t / 2, \mathcal{S}_{\pi}\right) \\
& \leq \mathbf{P}\left(\operatorname{Expo}\left(c_{k}\right)>t / 2\right) \mathbf{P}\left(\mathcal{S}_{\pi}\right)+\mathbf{P}\left(X_{k}>t / 2, \mathcal{S}_{\pi}\right)
\end{aligned}
$$

From the definition of the canonical end time $Z_{\pi}$, if $\mathcal{S}_{\pi}$ occurs, then $X_{k}=Z_{\pi}$. Therefore, we have

$$
\mathbf{P}\left(\mathcal{T}_{\pi \cdot t}\right) \leq \mathbf{P}\left(\operatorname{Expo}\left(c_{k}\right)>t / 2\right) \mathbf{P}\left(\mathcal{S}_{\pi}\right)+\mathbf{P}\left(Z_{\pi}>t / 2, \mathcal{S}_{\pi}\right) .
$$

Using Lemma 4.2, we can write

$$
\mathbf{P}\left(Z_{\pi}>t / 2, \mathcal{S}_{\pi}\right) \leq \frac{1}{(2 k) !}(t / 2)^{2 k-1} e^{-\beta t / 2} \beta^{k} \prod_{j=0}^{k-1} \frac{1}{c_{j}} .
$$

Meanwhile, from the exponential distribution and Lemma 4.1,

$$
\mathbf{P}\left(\operatorname{Expo}\left(c_{k}\right)>t / 2\right) \mathbf{P}\left(\mathcal{S}_{\pi}\right) \leq e^{-c_{k} t / 2} \beta^{k} \prod_{j=0}^{k-1} \frac{1}{c_{j}} \leq e^{-\beta t / 2} \beta^{k} \prod_{j=0}^{k-1} \frac{1}{c_{j}} .
$$

This implies

$$
\begin{aligned}
\mathbf{P}\left(\mathcal{T}_{\pi, t}\right) & \leq \mathbf{P}\left(\operatorname{Expo}\left(c_{k}\right)>t / 2\right) \mathbf{P}\left(\mathcal{S}_{\pi}\right)+\mathbf{P}\left(Z_{\pi}>t / 2, \mathcal{S}_{\pi}\right) \\
& \leq e^{-\beta t / 2}\left(1+\frac{1}{(2 k) !}(t / 2)^{2 k-1}\right) \beta^{k} \prod_{j=0}^{k-1} \frac{1}{c_{j}}
\end{aligned}
$$

For a path $\pi=\left(v_{0}, \ldots, v_{k}\right)$, we say that $\pi$ is safe if $c_{v_{i}} \geq d_{v_{i}}$ for $0 \leq i \leq k$.

We denote by $\mathcal{P}_{k}$ the set of paths originating in $S$ of length exactly $k$, and we define $\mathcal{P}_{k}^{\prime}$ correspondingly for safe paths. We will prove the following lemma, 
which states that the probability that an infection persists within the inoculated nodes can be made arbitrarily small by choosing an appropriate length of time.

Lemma 4.4. Suppose $G$ is a contact graph on $n$ nodes with seed set $S$ of size $s$. Then for any $h>0$ and any infection rate $\beta$,

$$
\mathbf{P}\left(\bigcup_{\pi \in \mathcal{P}^{\prime}} \mathcal{T}_{\pi}\right) \leq \sum_{\pi \in \mathcal{P}^{\prime}} \mathbf{P}\left(\mathcal{T}_{\pi}\right) \leq \frac{s h}{\beta(1-\beta)}
$$

if

$$
t \geq 8\left(\frac{\log \left(\frac{1}{h}\right)+\log (2 \beta)}{\min \left(\beta, \beta \log \left(\frac{1}{\beta}\right)\right)}\right)=c \log (1 / h)+c^{\prime}
$$

where $c$ and $c^{\prime}$ depend only on $\beta$ and not on $n$ or $s$.

Proof. Our strategy is to analyze short paths and long paths separately. For long paths, we will use Lemma 4.1, and for short paths, we will use Lemma 4.3.

Building on our definitions of $\mathcal{P}_{k}$ and $\mathcal{P}_{k}^{\prime}$, we define $\mathcal{P}_{\geq k}=\bigcup_{j=k}^{\infty} \mathcal{P}_{j}, \mathcal{P}_{\geq k}^{\prime}, \mathcal{P}_{<k}$, and $\mathcal{P}_{<k}^{\prime}$ accordingly. Let $k_{0}$ be the cutoff between long and short paths to be determined later. For paths of length at least $k_{0}$, we observe that

$$
\begin{aligned}
\sum_{\pi \in \mathcal{P}_{\geq k_{0}}^{\prime}} \mathbf{P}\left(\mathcal{I}_{\pi}\right) & \leq \sum_{k=k_{0}}^{\infty} \sum_{\pi \in \mathcal{P}_{k}^{\prime}} \mathbf{P}\left(\mathcal{S}_{\pi}\right) \\
& \leq \sum_{k=k_{0}}^{\infty} \sum_{v_{0} \in S} \sum_{v_{1} \sim v_{0}} \ldots \sum_{v_{k} \sim v_{k-1}} \mathbf{P}\left(\mathcal{S}_{\left(v_{0}, \ldots, v_{k}\right)}\right) \\
& \leq \sum_{k=k_{0}}^{\infty} \sum_{v_{0} \in S} \sum_{v_{1} \sim v_{0}} \ldots \sum_{v_{k} \sim v_{k-1}} \beta^{k} \prod_{j=0}^{k-1} \frac{1}{c_{j}} \\
& \leq \sum_{k=k_{0}}^{\infty} \sum_{v_{0} \in S} \sum_{v_{1} \sim v_{0}} \ldots \sum_{v_{k} \sim v_{k-1}} \beta^{k} \prod_{j=0}^{k-1} \frac{1}{d_{j}} \\
& =\frac{s \beta^{k_{0}}}{1-\beta} .
\end{aligned}
$$


On the other hand, for paths of length less than $k_{0}$, we have

$$
\begin{aligned}
\sum_{\pi \in \mathcal{P}_{<k_{0}}^{\prime}} \mathbf{P}\left(\mathcal{T}_{\pi}\right) & \leq \sum_{k=0}^{k_{0}-1} \sum_{\pi \in \mathcal{P}_{k}^{\prime}} \mathbf{P}\left(\mathcal{T}_{\pi}\right) \\
& \leq \sum_{k=0}^{k_{0}-1} \sum_{v_{0} \in S} \sum_{v_{1} \sim v_{0}} \cdots \sum_{v_{k} \sim v_{k-1}} \mathbf{P}\left(\mathcal{T}_{\pi}\right) \\
& \leq \sum_{k=0}^{k_{0}-1} \sum_{v_{0} \in S} \sum_{v_{1} \sim v_{0}} \cdots \sum_{v_{k} \sim v_{k-1}} e^{-\beta t / 2}\left(1+\frac{1}{(2 k) !}\left(\frac{t}{2}\right)^{2 k-1}\right) \beta^{k} \prod_{j=0}^{k-1} \frac{1}{c_{j}} \\
& \leq s \sum_{k=0}^{k_{0}-1} e^{-\beta t / 2} \beta^{k}\left(1+\frac{\left(\frac{t}{2}\right)^{2 k-1}}{(2 k) !}\right) \\
& \leq s k_{0} e^{-\beta t / 2}\left(1+\frac{\left(\frac{t}{2}\right)^{2 k_{0}-1}}{\left(2 k_{0}\right) !}\right)
\end{aligned}
$$

Combining the bounds for short and long paths yields

$$
\sum_{\pi \in \mathcal{P}^{\prime}} \mathbf{P}\left(\mathcal{T}_{\pi}\right) \leq s\left(\frac{\beta^{k_{0}}}{1-\beta}+k_{0} e^{-\beta t / 2}\left(1+\frac{\left(\frac{t}{2}\right)^{2 k_{0}-1}}{\left(2 k_{0}\right) !}\right)\right) .
$$

We choose $k_{0}=\beta t / 8$. Because $t \geq 8 / \beta$, we can use Stirling's approximation to derive the bound

$$
e^{\beta t / 4} \geq k_{0}+\frac{\left(\frac{t}{2}\right)^{2 k_{0}}}{\left(2 k_{0}\right) !}
$$

Thus we have

$$
\sum_{\pi \in \mathcal{P}^{\prime}} \mathbf{P}\left(\mathcal{T}_{\pi}\right) \leq s\left(\frac{\beta^{k_{0}}}{1-\beta}+e^{-\beta t / 4}\right) \leq s\left(\frac{\beta^{\beta t / 8}}{1-\beta}+e^{-\beta t / 4}\right) \leq \frac{s h}{\beta(1-\beta)}
$$

from the assumption

$$
t \geq 8\left(\frac{\log \left(\frac{1}{h}\right)+\log (2 \beta)}{\beta \log \left(\frac{1}{\beta}\right)}\right), \quad \beta^{\beta t / 8} \leq \frac{s h}{2 \beta(1-\beta)} .
$$

This completes the proof of Lemma 4.4. 


\section{Proof of the Main Theorems}

We are now ready to prove Theorem 3.1. Suppose that an infection starts in $S \subseteq H \subseteq V$, and each node $v \in H$ is inoculated with $c_{v}=d_{v}$. Recall that $\mathcal{E}_{H}$ denotes the event that an infection started in $S$ ever leaves the set $H$. Theorem 3.1 states that $\mathcal{E}_{H}$ satisfies

$$
\mathbf{P}\left(\mathcal{E}_{H}\right) \leq \frac{s}{\beta} \operatorname{pr}\left(1-\beta, \frac{\mathbf{1}_{S}}{s}\right) \mathbf{1}_{\bar{H}}^{*} .
$$

Proof of Theorem 3.I. Let $\mathcal{B}_{k}$ denote the set of all paths of length $k$ from $S$ to $\bar{H}$ such that the first $k-1$ steps are in $H$. We define $\mathcal{B}$ to be the union of all $\mathcal{B}_{k}$. Note that if $u \in \bar{H}$ is ever infected, then $\mathcal{S}_{\pi}$ occurs for some $\pi \in \mathcal{B}$. We will bound that probability using the union bound:

$$
\begin{aligned}
\sum_{\pi \in \mathcal{B}} \mathbf{P}\left(\mathcal{S}_{\pi}\right) & \leq \sum_{k} \sum_{\pi \in \mathcal{B}_{k}} \mathbf{P}\left(\mathcal{S}_{\pi}\right) \\
& \leq \sum_{k} \sum_{v_{0} \in S} \sum_{v_{k} \in \bar{H}} \sum_{\pi=\left(v_{0}, \ldots, v_{k}\right) \in \mathcal{B}_{k}} \mathbf{P}\left(\mathcal{S}_{\pi}\right) \\
& \leq \sum_{k} \sum_{v_{0} \in S} \sum_{v_{k} \in \bar{H} \pi=\left(v_{0}, \ldots, v_{k}\right) \in \mathcal{B}_{k}} \beta^{k} \prod_{j=0}^{k-1} \frac{1}{d_{j}} \\
& =\sum_{k} \mathbf{1}_{S} \beta^{k}\left(D^{-1} A\right)^{k} \mathbf{1}_{\bar{H}}^{*} \\
& =\sum_{k} \mathbf{1}_{S} \beta^{k} W^{k} \mathbf{1}_{\bar{H}}^{*} \\
& =\frac{s}{\beta} \operatorname{pr}\left(1-\beta, \frac{\mathbf{1}_{S}}{s}\right) \mathbf{1}_{\bar{H}}^{*}
\end{aligned}
$$

where we use (2.1) and the assumption on $H$, and apply Lemma 4.1.

Note that if $\pi$ is a path that contains vertices in $\bar{H}$, then it has an initial segment $\bar{\pi} \in \mathcal{B}$, and $\mathcal{S}_{\pi} \subseteq \mathcal{S}_{\bar{\pi}}$. The set of such walks is $\mathcal{P} \backslash \mathcal{P}^{\prime}$; we have shown that

$$
\mathbf{P}\left(\bigcup_{\pi \in \mathcal{P} \backslash \mathcal{P}^{\prime}} \mathcal{T}_{\pi}\right)=\mathbf{P}\left(\bigcup_{\pi \in \mathcal{B}} \mathcal{T}_{\pi}\right) \leq \sum_{\pi \in \mathcal{B}} \mathbf{P}\left(\mathcal{S}_{\pi}\right) \leq \frac{s}{\beta} \operatorname{pr}\left(1-\beta, \frac{\mathbf{1}_{S}}{s}\right) \mathbf{1}_{\bar{H}}^{*}
$$

Thus, we have shown that the probability that the infection leaves $H$ depends on the personalized PageRank on $\bar{H}$.

We are now ready to prove our next theorem. 
Proof of Theorem 3.2. By the assumptions, $H$ is a cluster with $S$ contained in its $(1-\beta)$-core. Thus, for $u \in S$, we have from $(2.3)$,

$$
\operatorname{pr}\left(1-\beta, \mathbf{1}_{u}^{*}\right) \mathbf{1}_{\bar{H}}^{*} \leq \frac{h}{1-\beta}
$$

Summing over all $u \in S$ gives

$$
\operatorname{pr}\left(1-\beta, \frac{\mathbf{1}_{S}}{s}\right) \mathbf{1}_{\bar{H}}^{*}=\frac{1}{s} \sum_{u \in S} \operatorname{pr}\left(1-\beta, \mathbf{1}_{u}\right) \mathbf{1}_{\bar{H}}^{*} \leq \frac{h}{1-\beta} .
$$

Applying this bound to Theorem 3.1 gives

$$
\mathbf{P}\left(\mathcal{E}_{H}\right) \leq \frac{s h}{\beta(1-\beta)} .
$$

Thus, the probability that the infection escapes $H$ is at most $\frac{s h}{\beta(1-\beta)}$. Because $c_{i}=d_{i}$ for $v_{i} \in H$, all paths within $H$ are safe paths, and we can apply Lemma 4.4 to bound the probability that the infection persists in $H$. Lemma 4.4 implies that the probability that the infection persists in $H$ for longer than $c \log \left(\frac{1}{h}\right)+c^{\prime}$ time is also at most $\frac{s h}{\beta(1-\beta)}$. Combining these two results yields that the probability that the infection persists anywhere on the contact graph for longer than $c \log s+c^{\prime}$ time is at most $2 \frac{s h}{\beta(1-\beta)}$.

Theorem 3.4 implies that if $S$ is chosen randomly from an $h$-cluster $H$, then there is a high probability that it is also in the core of $H$. This is important, because if $S$ is in the core of $H$, then we can effectively combat any infection starting from $S$ by inoculating only $H$. The proof is similar to the analysis involved in local partitioning algorithms using PageRank [Andersen et al. 06].

Proof of Theorem 3.4. Suppose we are given an $h$-cluster $H$, and $S$ is formed by selecting $s$ random vertices from $H$, independently with probability proportional to their degrees. Suppose $v$ is one of those $s$ nodes, and let $X$ be a random variable that marks the amount of personalized PageRank contained in $\bar{H}$ :

$$
X=\operatorname{pr}\left(\alpha, \mathbf{1}_{v}\right) \mathbf{1}_{\bar{H}}^{*}
$$

From [Andersen et al. 06], we have

$$
\mathbf{E}(X) \leq \frac{h}{2 \alpha}
$$

where the expectation is over the possible nodes $v \in H$. Furthermore, since $X \leq 1$, we can bound the variance by $\operatorname{Var}(X) \leq \mathbf{E}(X)$. 
Here we take $\alpha=1-\beta$. Since we are selecting $s$ random vertices, we consider $Y=\sum_{i=1}^{s} X_{i}$, where $X_{i}$ is a copy of $X$. We are interested in bounding

$$
\mathbf{P}\left(Y \geq \frac{s h}{\alpha}\right) \leq \mathbf{P}(Y \geq 2 \mathbf{E}(Y)) .
$$

Using Chernoff's inequality and the known bound for $\mathbf{E}(X)$, we have

$$
\mathbf{P}(Y \geq 2 s \mathbf{E}(X)) \leq e^{-s \mathbf{E}(X)^{2} /(2 \operatorname{Var}(X))} \leq e^{-s h /(4 \alpha)} \leq \epsilon,
$$

since $\operatorname{sh} /(4 \alpha) \geq \log (1 / \epsilon)$.

By Theorem 3.1, with probability at most $1-\epsilon$, the event that the infection starting from $S$ leaves $H$ satisfies

$$
\mathbf{P}\left(\mathcal{E}_{H}\right) \leq \frac{s}{\beta} \operatorname{pr}\left(1-\beta, \frac{\mathbf{1}_{S}}{s}\right) \mathbf{1}_{\bar{H}}^{*} \leq \frac{s h}{4 \alpha \beta} \leq \frac{s h}{4(1-\beta) \beta} \leq \epsilon,
$$

by the assumption $s h \leq \epsilon$. This completes the proof of Theorem 3.4.

\section{Concluding Remarks}

There are many questions remaining, several of which we mention here:

1. In this paper, we show that if $s$ infected nodes are in the core of an $h$-cluster $H$ and the product of $s$ and $h$ is small, then we need only inoculate nodes in $H$ so that the infection will die out in $O(\log s)$ time with high probability. Is it possible to improve or replace the condition imposed on the product $s h$ ?

2. In our main theorems, our analysis involves the Cheeger ratio, which is one of the parameters concerning the structure of a graph. It will be desirable if other structural parameters can help improve the probabilistic bounds in the statement of Theorem 3.2, for example.

3. In this paper, we consider a fixed infection rate $\beta$ and ask how little antidote can be used while still ensuring that the contact process dies out quickly. The other natural approach to this problem is to fix an amount of antidote and ask for what range of $\beta$ will the disease necessarily die out quickly.

4. One can also consider alternative models of contact process in which cured nodes may or may not be susceptible to reinfection. In addition, the type of propagation on networks can be different.

Many related interesting questions remain to be answered. 
Acknowledgments. The first author's research has been supported in part by ONR MURI N000140810747 and AF/SUB 552082.

\section{References}

[Andersen and Chung 07] R. Andersen and F. Chung. "Detecting Sharp Drops in PageRank and a Simplified Local Partitioning Algorithm." In Theory and Applications of Models of Computation: 4th International Conference, TAMC 2007, Shanghai, China, May 22-25, 2007, Proceedings, Lecture Notes in Computer Science 4484, edited by Jin-Yi Cai, S. Barry Cooper, and Hong Zhu, pp. 1-12. Berlin: Springer, 2007.

[Andersen et al. 06] R. Andersen, F. Chung, and K. Lang. "Local Graph Partitioning Using PageRank Vectors." In Proceedings of the 47th Annual IEEE Symposium on Foundation of Computer Science, pp. 475-486. Washington, DC: IEEE Computer Society, 2006.

[Beger et al. 05] N. Berger, C. Borgs, J. Chayes, and A. Saberi. "On the Spread of Viruses on the Internet." In Proceedings of the 16th Annual ACM-SIAM Symposium on Discrete Algorithms, pp. 301-310. Philadelpia: SIAM, 2005.

[Borgs et al. 10] C. Borgs, J. Chayes, A. Ganesh, and A. Saberi. "How to Distribute Antidote to Control Epidemics." To appear in Random Structures and Algorithms, 2010 .

[Brin and Page 98] S. Brin and L. Page. "The Anatomy of a Large-Scale Hypertextual Web Search Engine." Computer Networks and ISDN Systems 30 (1998), 107-117.

[Chen and Carley 03] L. Chen and K. Carley. "The Impact of Network Topology on the Spread of Anti-virus Countermeasures." Paper presented at NAACSOS 2003 Conference, Pittsburgh, PA, June 22-25, 2003.

[Dezso and Barabasi 02] Z. Dezso and A. Barabasi. "Halting Viruses in Scale-Free Networks." Physical Review E 65 (2002), 055103.

[Forster and Gilligan 07] G. Forster and C. Gilligan. "Optimizing the Control of Disease Infestations at the Landscape Scale. Proceedings of the National Academy of Sciences 104 (2007), 4984-4989.

[Ganesh et al. 05] A. Ganesh, L. Massoulie, and D. Towsley. "The Effect of Network Topology on the Spread of Epidemics." In Proceedings of the 24th Annual Joint Conference of the IEEE Computer and Communications Societies (INFOCOM 2005), Vol. 2, pp. 1455-1466. Los Alamitos, CA: IEEE Press, 2005.

[Kermack and McKendric 27] W. Kermack and A. McKendric. "A Contribution to the Mathematical Theory of Epidemics." Proceedings of the Royal Society of London A 115 (1927), 700-721.

[Kiss et al. 05] I. Kiss, D. Green, and R. Kao. "Infections Disease Control Using Contact Tracing in Random and Scale-Free Networks." Journal of the Royal Society Interface 3 (2005), 55-62. 
[May and Lloyd 01] R. May and A. Lloyd. "Infection Dynamics on Scale-Free Networks." Physical Review E 64 (2001), 066112.

[Miller and Hyman 07] J. Miller and J. Hyman. "Effective Vaccination Strategies for Realistic Social Networks." Physica A 386 (2007), 780-785.

[Newman 02] M. Newman. "The Spread of Epidemic Disease on Networks." Physical Review E 66 (2002), 016128.

[Pastor-Sattoras and Vespignani 01] R. Pastor-Sattoras and A. Vespignani. "Epidemic Spreading in Scale-Free Networks." Physical Review Letters 86 (2001), 3200-3203.

[Pastor-Sattoras and Vespignani 02] R. Pastor-Sattoras and A. Vespignani. "Epidemic Dynamics in Finite Size Scale-Free Networks." Physical Review E 65 (2002), 035108.

[Tsimring and Huerta 03] L. Tsimring and R. Huerta. "Modeling of Contact Tracing in Social Networks." Physica A 325 (2003), 33-39.

Fan Chung, University of California, San Diego, Department of Mathematics, 9500 Gilman Drive, La Jolla, CA 92093-0112 (fan@math.ucsd.edu)

Paul Horn, Emory University, Department of Mathematics and Computer Science, 400 Dowman Dr., W401, Atlanta, GA 30322 (phorn@mathcs.emory.edu)

Alexander Tsiatas, University of California, San Diego, Department of Computer Science and Engineering, 9500 Gilman Drive, La Jolla, CA 92093-0404

(atsiatas@cs.ucsd.edu)

Received February 21, 2010; accepted May 11, 2010. 